\title{
DOCUMENTATION
}

\section{La Pompe centrifuge et ses Applications.}

\author{
Par COM'TE. Ingènieur.
}

\begin{abstract}
Dès la plus haute antiquité, les hommes ont eu à résoudre le problème du transport dé l'eall d'ù̀ lieu à un autre. N'existe-t-il pas en France un grand nombre de vestiges proubant que les Romains, par exemple, se préoccupaient déjà du transport de l'eau par aqueducs pour alimenter leurs somptueuses piscines?

A u cours de ce dernier siècle, les besoins en eau se sont fait sentir dans tous les domaines et ci. liquide est devenu absolument indispensable à notre civilisation.

Les moyens mis en usage au cours des siècles sont bien différents et hous ne destrons nullement les énumérer ici, mais seulement attirer l'attention du lecteur et de l'industriel sur celle question du transport des liquides en général el souligner les avantages principaux que présente la pompe centrifuge.
\end{abstract}

Les applications de l'électro-mécanique qui, depuis de nombreuses années, ont pris une extension prodigieuse, ont beaucoup influencé le développement et l'application de la pompe centrifuge; on peut même dire que certaines créations de la mécanique moderne résultent de l'influence de l'électro-technique. corps de pompe, saisit le liquide et le déplace sous l'action de la force centrifuge. La forme des tubes varie avec la nature du travail que la pompe est chargée d'accomplir.

On distingue trois types de pompes centrifuges : basse, moyenne et haute pression. Elles peuvent être à axe ho. rizontal ou à axe vertical. Les pompes centrifuges à haute

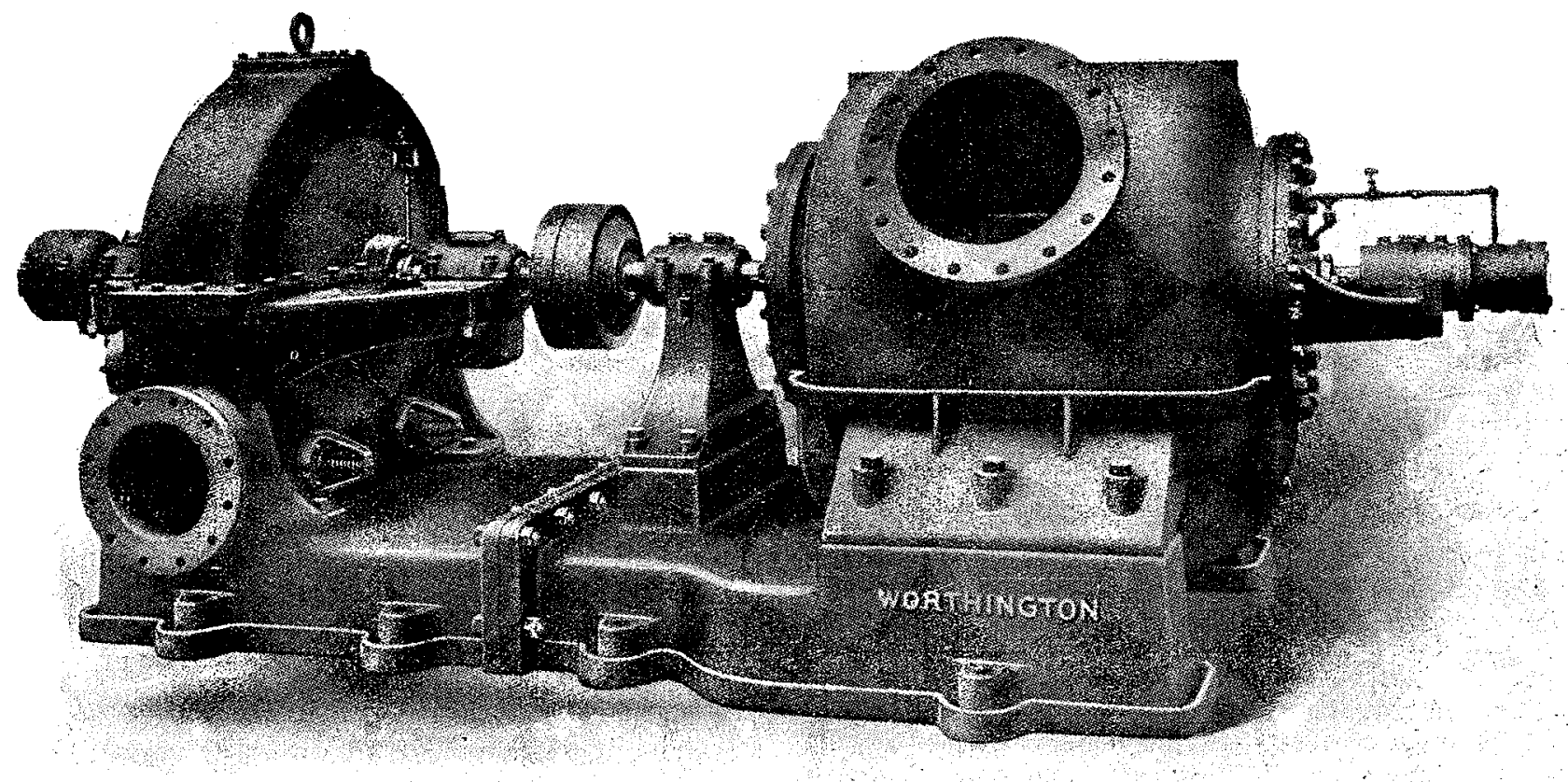

Pompe centrifuge " Worthington

Type de cireulation clirectement actionnée par lurbine à vapeur.

On sait que les machines électriques sont celles qui conviennent le mieux à l'entraînement direct des machines rotätives. Or, la transmission électrique étant, pour des raisons qu'il serait superflu d'exposer ici, supérieure à toutes les autres transmissions de force, il est tout naturel que les industriels tendent à créer dans la mesure du possible, des machines rotatives.

Les progrès qui ont été réalisés ces dernières années dans la construction des moteúrs électriques à grande vitesse (3.000 tours par minute pour courant triphasé, 50 périodes et pour des puissances de plusieurs centaines de chevaux effectifs) ont permis d'augmenter encore le champ d'application déjà vaste des pompes centrifuges.

Les pompes centrifuges se composent de deuxx parties principales quii sont : le corps de pompe et la roue à aubes, qu'on appelle aussi impulseur. L'impulseur tourne dans le pression diffèrent de celles à basse pression par un appareil directeur, appelé diffuseur, et qui a l'avantage d'angmenter sensiblement leur rendement utile.

A la sortie de l'impulseur l'eau, animée de sa vitesse maxima, est reçue par ce diffuseur, dont la forme est itgoureusement établie en fonction de l'angle de sortie dè l'eau. Cet appareil est constitué par des canaux à sections croissantes. La réaction du liquide sur les aubes fixes achève de transformer la vitesse en pression hydraulique.

De la construction soignée de cet appareil et de la trans. formation rationnelle de la vitesse en pression, dépend justement le rendement élevé des pompes à haute pression:

L'emploi du diffuseur a permis d'utiliser des pompes un seul impulseur pour des hauteurs d'élévation de plus de 100 mètres et d'obtenir un rendement supérieur. 


\section{Pompes centrifuges a Basse Pression}

La figure ci-dessous représente une pompe à basse pression employée comme pompe de circulation d'un condenseur, entraînée directement par une turbine à vapeur.

Ces pompes à basse pression trouvent également leur application dans les travaux publics, alimentation générale, irrigation, assèchement des cales de radoub, etc... La figure No 7035 représente une telle pompe à axe vertical.

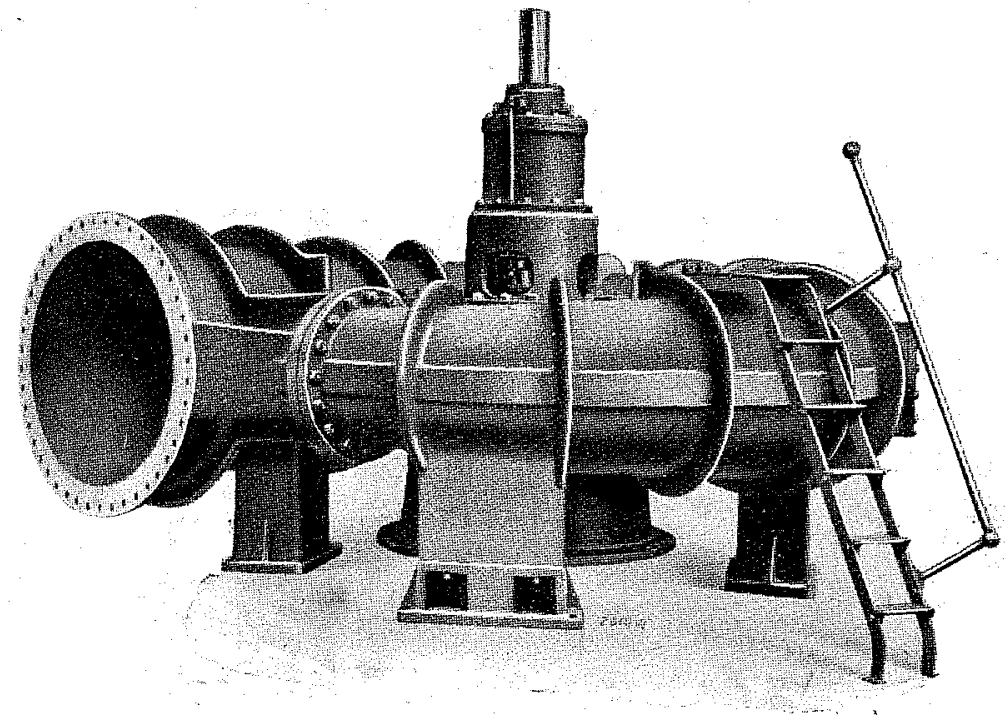

Pompe centrifuge "Worthington "

Type verticale de $1 \mathrm{~m} .370$ d'orifice pour épuisement des cales de radoub.

Elles sont généralement employées pour tous débits et pour des hauteurs d'élévation de 1 à 20 mètres. Ces appareils ne possèdent qu'un impulseur et sont de construction simple et robuste. Leur conception hydraulique est très soignée et permet d'obtenir des rendements supérieurs. $(72$ à $80 \%$ ) ; leur puissance d'aspiration est très grande.

\section{Pompes centrifuges a moyenne Pression}

Ce type de pompes possède, de même que le type à basse pression, un seul impulseur. Toutefois, comme son

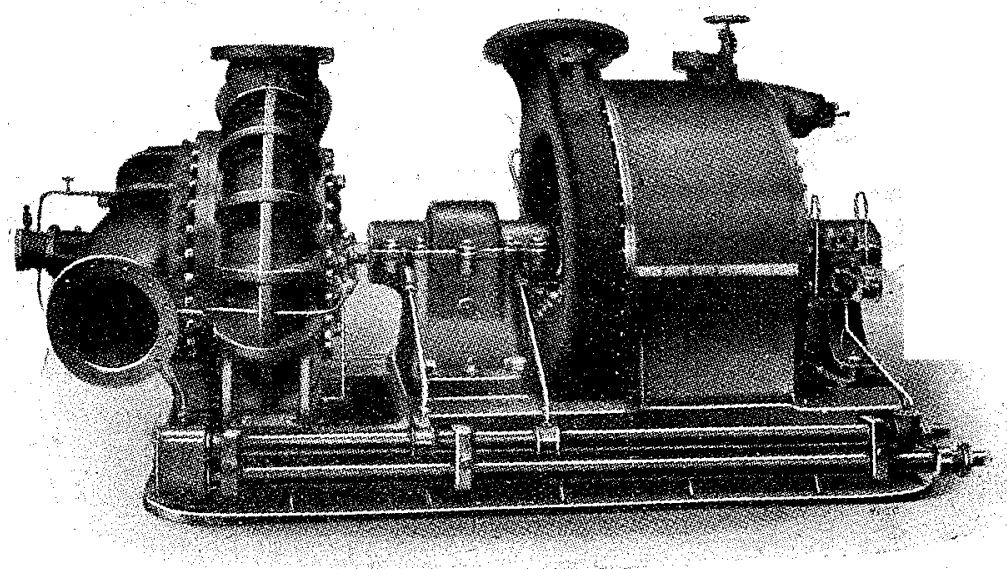

Groupe Turbo. Pompe "Worthington" Type marine d'incendie.

nom l'indique, la pression de régime varie de 10 à 50 mètres. Il est avantageusement employé dans l'alimentation des châteaux d'eau, comme pompe à incendie à poste fixe ou mobile, etc... La figure No 7034 montre une pompe à moyenne pression entraînée directement par une turbine à vapeur, groupe qui est destiné au service d'incendie pour paquebots.

Ces pompes peuvent être entraînées par courroie ou directement par moteurs électriques, machines à vapeur, turbines à vapeur, moteurs à essence ou huile lourde ; voire même dans certains cas par turbines hydrauliques.

\section{Pompes centrifuges a haute Pression}

Ces pompes différent des pompes à basse et moyenne pression, non seulement par le diffuseur, mais par le nombre de segments qui la composent. En effet, comme le nom même l'indique, l'on peut obtenir avec ces pompes des pressions allant jusqu'à 600 et 700 mètres. Ces hauteurs considérables s'obtiennent en plaçant les.uns à còté des autres et sur le même arbre plusieurs impulseurs, à chacun desquels correspond un diffuseur et un appareil directeur.

Le liquide qui-sort du premier impulseur sous une pression déterminée est amené par l'appareil directeur à l'entrée du deuxième impulseur. Il sort du deuxième impulseur sous une pression double. Du second impulseur le liquide passe dans le troisième, le quatrième, le n-ième. La pression finale sera trois, quatre, n-fois la pression obtenue par un seul impulseur.

Les différents éléments qui forment ces diffuseurs et appareils directeurs, qu'on appelle pièces intermédiaires, sont assemblés et fixés entre le couvercle d'aspiration et le couvercle de refoulement. Ces deux dernières pièces sont pourvues de fortes brides sur lesquelles viennent se fixer les boulons d'assemblage (voir figure ci-dessous).

Le nombre d'impulseurs et de segments qu'il convient d'adopter pour constituer la pompe, suivant la pression totale qu'elle doit engendrer, dépend de diverses circonstances techniques, notamment de la parfaite exécution des organes en mouvement et de la bonne construction des paliers. L'expérience acquise dans ce domaine fixe ce nombre à 8 et 10 au maximum. suivant la grandeur de la pompe.

Lorsque la pression totale demandée, divisée par la pression de chaque impulseur qui est limitée, nous donne un chiffre supérieur. à 8 ou 10, l'on constitue ce qu'on appelle un groupe de deux pompes centrifuges marchant en série. Le moteur est généralement placé entre les deux pompes, qui sont reliées entre elles par une conduite intermédiaire.

Les rendements des pompes à haute pression sont élevés et avec les grands modèles l'on peut facilement atteïndre 72 à $78 \%$ et plus, suivant les conditions d'élévation. La hauteur d'élévation maximum qui est généralement admise par impulseur à haute pression est de 60 mètres.

Une des caractéristiques principales de la pompe centrifuge est qu'on peut en pleine exploitation fermer complètement la conduite de refoulement, sans aucun danger pour la pompe elle-même et pour ses organes. L'emploi de ces pompes centrifuges est donc tout indiqué pour l'alimentation de châteaux d'eau avec réglage par flotteur, pour l'alimentation de chaudières (voir figure $N^{\circ} 7052$ ) et des accumulateurs de pression.

Le champ d'application des pompes centrifuges à haute pression est très vaste. Elles sont actuellement employées comme pompes d'épuisement, de mines (jusqu'à 600 mètres de profondeur et plus), pour. l'alimentation en eau potable des villes, pour la mancuvre hydraulique des engins de ports, etc... 
Les travaux de fonçage des mines se font aussi au moyen de pompes centrifuges et la figure cj-desscus nous montre un type spécial à axe vertical

La pompe est fixée à un cadre en fers profilés. Le moteur électrique est disposé directement sur la pompe. A la partie supérieure du cadre est fixée une poulie à gorges autour de laquelle vient s'enrouler le câble de suspension.

\section{Conduttes d'Aspiration et de Refoulement}

La hauteur d'aspiration qui peut être obtenue avec les pompes centrifuges est de 8 mètres (eau froide). L'étanchéité de la conduite d'aspiration doit être parfaite. Les presse-étoupe doivent empêcher toute infiltration d'air dans le corps de pompe.

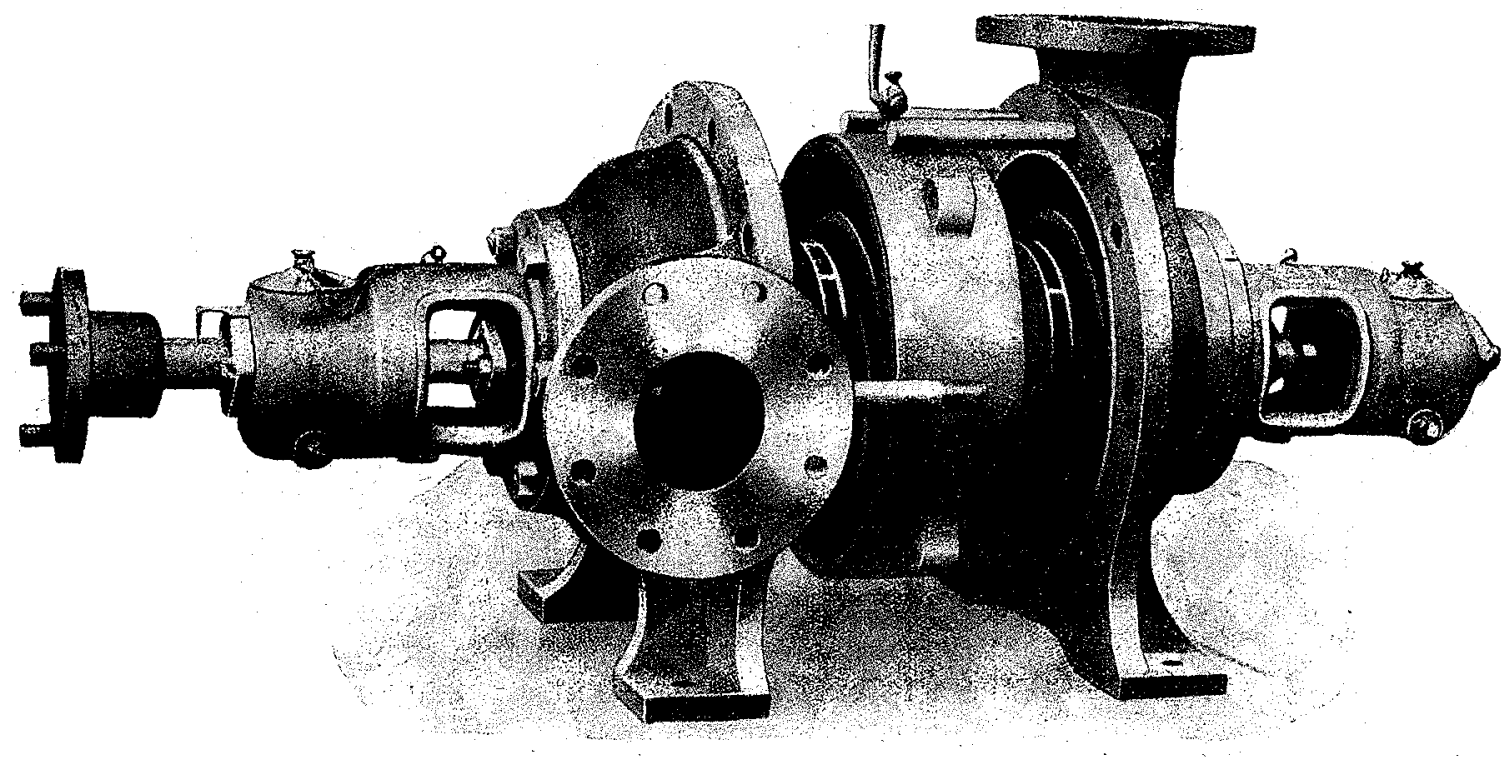

Pompe centifuge "Worthington sà haute pression.

Lè groupe ainsi constitué est descendu au fond du puits jusqu'à la zone de fonçage. La colonne de refoulement est généralement fixée directement dans l'axe mème du groupe moto-pompe. Le groupe continue à descendre au für et à mesure de l'épuisement des eaux d'infiltration. Ces pompes sont généralement prévues pour des hauteurs ne dépassant pas 150 mètres.
On a souvent rèproché aux pompes centrifuges l'inféirorité d'aspiration par rapport aux pompes à piston. Ce reproche ne serait pas fondé si l'on avait voué plus d'attention

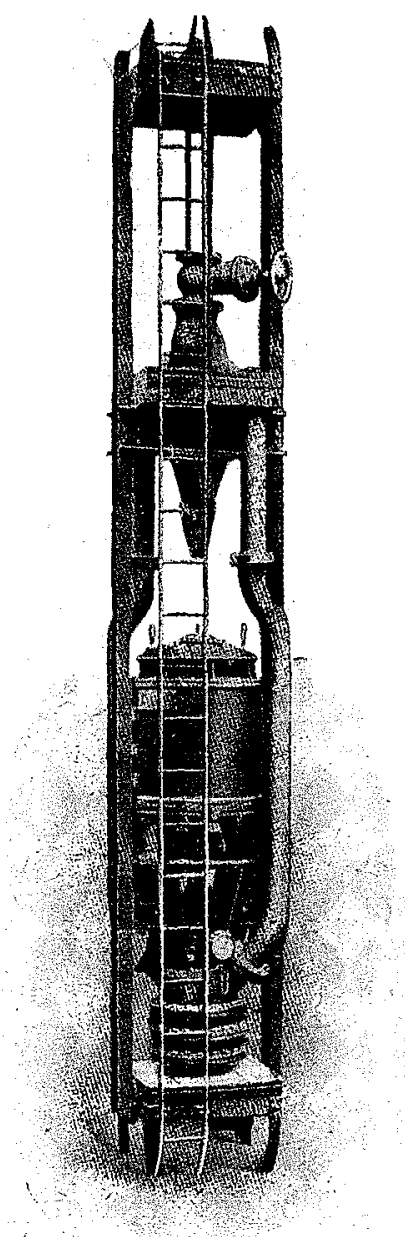

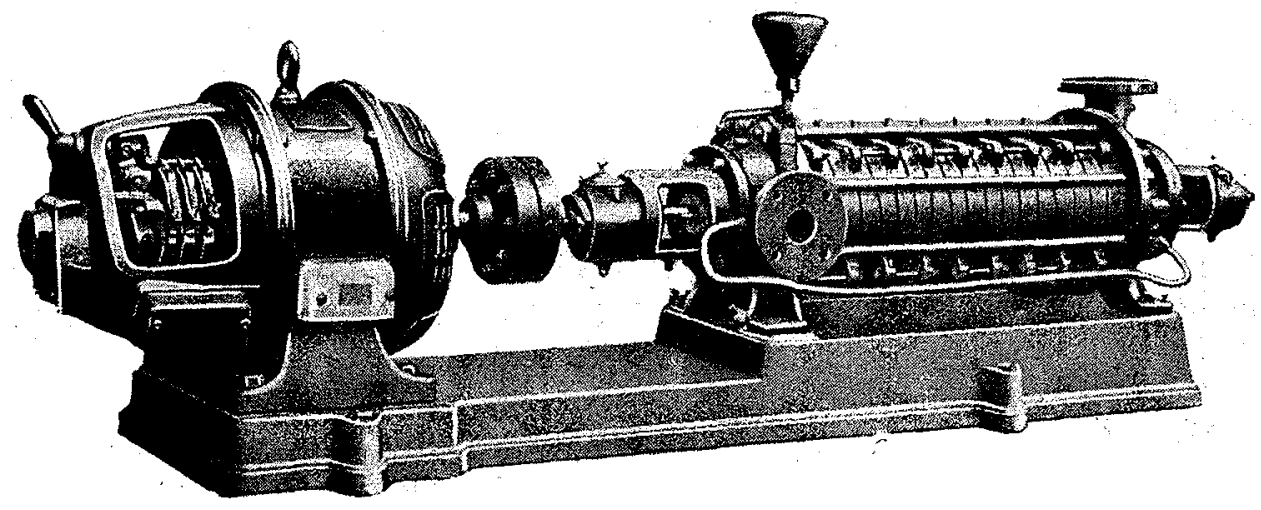

Groupe moto. Pompe centrifuge "Worthington "à haute pression pour alimentation de chaudieres.
Pompe centrifuge Worthingtou Type de fonçage.

à ces questions d'étanchéité. Il a été établi des conduites d'aspiration d'une longueur de plus de 1.000 mètres sans qu'il en soit résulté aucun inconvénient.

Il faut surtout prendre soin qu'il ne puisse se former de poches d'air et que tous les joints soient bien étanches. Enfin, il convient de donner à la conduite une légère inclinaison, pour permettre aux bulles d'air qui se dégagent toujours de l'eau, d'être évacuées par la pompe centrifuge.

Pour le fonçage ou l'épuisement de mines plus profondes, l'on installe à mi-hauteur un ou plusieurs groupes fixes à axe horizontal qui refoulent alors jusqu'au jour.

Grâce à l'espace très restreint nécessité par ces pompes, il reste assez de place pour le passage des bennes. Les avantages que présentent cés pompes de fonçage expliquent leur emploi de plus en plus étendu, car elles assurent aux exploitations minières un puissant auxiliaire.
L'établissement des conduites de refoulement ne présente aucune difficulté. Il n'est pas nécessaire de prévoir des réservoirs d'air, puisque le mouvement imprimé à l'eau parla pompe centrifuge est absolument continu. Le passage de l'eau de la conduite d'aspiration à la conduite de refoulement se fait sans choc et il n'y a aucune instabilite dans la colonne d'eau en mouvement. 


\section{Utilisation des Combustibles.}

Nous croyons intéressant de signaler aux lecteurs de lat Houille Bhthche, le Rapport de M. Rauber, à la Commisșion d'utilisation des combustibles. Cette étude constitue un document de premier ordre pour tous ceux 'qui s'occupent d'exploitation et d'Installations de Centrales à vapeur chauffées au charbon.

Après avoir énuméré les caractéristiques d'une Centrale, l'auteur envisage les différentes solutions, et sortes de matériel employées.

Types de chaudières, Surchauffeurs, Economiseurs, Réchauffeurs d'air, Tirage, Foyers, Accessoires divers des chaudières, Tuyauterie de vapeur, Eau d'alimentation, Conduite des chaufferies, Traitement des mâchefers, Turbines à vapeur, Condensation et - Alternateurs.

M. Rauber termine son travail par les conclusions suivantes :

I. - Une centrale à vapeur moderne, petite ou grande, est nécessairement un ensemble assez compliqué, dont on ne devra décider la création qu'après étude approfondie.

II. - En raison de cette complexité et de la rapidité d'évolution de la technicue, 11 est prudent de consulter, pour les études de centrales, des spécialistes éprouvés, afin d'être sûr de réaliser, dans chaque cas particulier, l'usine la moins chère comme établissement, la jJus moderne et la plus économique.
A ce point de vue on peut clésirer que réussissent, dans notre pays, des bureaux d'études de centrales spécialisées, comme il en existe en Angleterre et en Amérique, qui constituent par leur expérience un élément sérieux de perfectionnement.

III. - De grands progrès sont ericore possibles en ce qui concerne l'économie de combustible. Parmi les principaux citons :

I'emploi de pressions de vapeur plus élevées ;

L'emploi d'une forte surchauffe ;

I.e réchauffage de l'eau d'alimentation, notamment par des prélevements de'vapeur aux turbines ; dues.

Il est permis d'espérer, de l'application plus ou moins complète de ces perfectionnements, une économie de combustible de 20 à $40 \%$ par rapport à celle des bonnes centrales actuelles, toutes choses égales. Il s'impose que l'on soit, en France, ou la production des mines de combustible est inférieure aux hesoins du pays, à la tête du progrès dans cette voie.

IV. - Il est désirable, pour favoriser ce mouvement, que l'Etat et les grands groupements industriels provoquent et subventionment res travaux des physiciens et des savants en vue de poursuivre l'étude expérimentale des propriétés de la vapeur d'cau juscu'aux pressions et aux températures de surchauffe les plus élevées.

\section{Conférence internationale des Grands Réseaux de Transport d'énergie électrique à haute tension.}

La Conférence internationale des grands Réseaux de Transport d'énergie électrique à très haute tension, qui a eu lieu à Paris du 21 au 26 novembre 1921, au siège de l'Union des Syndicats de l'Electricité, 7 , rue de Madrid, a complètement atteint les buts que s'était proposés l'Union des Syndicats de l'Electricité, en organisant cette conférence. Les nombreux délégués français et étrangers ont adressé à diverses reprises au président de l'Union des Syndicats d'Electricité, M. Raynald Legonez, et à son secrétaire général, $M$. Tribot Laspière, leurs remerciements chaleureux pour la parfaite organisation de la Conférence..

Les délégués ont unanimenent exprimé le vœu que cette première Conférence soit suivie à bref délai d'une nouvelle réunion où seraient examinés certains points, qui n'ont pu être qu'effleurés au cours de la première réunion.

Un autre vœu, qu'il convient de signaler, est que les travaux de la Conférence internationale soient officiellement communiqués à la Commission électrotechnique internationale, en vue d'en tirer les conclusions. utiles concernant le travail d'unification dont est spécialement chargée cette commission.

Les délégués étrangers n'ont pas voulu se séparer sans envoyer leur salut à $M$. Blondel, qui, dès l'ouverture de la Conférence, avait été acclamé président d'honneur conjointement à M. Mailloux et que son état de santé a empêché d'assister aux séances; ils lui ont adressé l'expression unanime de leur sympathie et de leur admiration pour l'importance de ses travaux.

Le $\mathrm{D}^{\mathrm{r}}$ Mailloux, président de la Commission électrotechnique internationale, avait fait le voyage des Etats-Unis a Paris pour, en même temps qu'assister aux réunions de la Conférence, prendre la parole au cours de la cérémonie organisée en l'honneur d'Ampère, qui a eu lieu le jeudi 21 novembre, dans le grand amphithéâtre de la Sorbonne, sous la haute présidence de M. Millerand, président de la République française.

\section{Lampe Mazda demi-watt et la contrefaçon.}

La lampe connue de tous nos lecteurs sous le nom de lampe demi watt a pour origine, ainsi qu'on le sait, une invention due au docteur Langmuir, dont les brevets sont exploités par la General Electric Company et ses Filiales en tous pays (La Cie des Lampes en France).

La contrefaçon du brevet fondamental vient d'être l'objet de deux importants procès: I'un engagé aux Etats-Unis par la General Electric Company contre la Nitro-Tungsten Lamp $C^{0}$, et l'autre en Angleterre par la British Thomson-Houston Company litd contre la Corona Lamp Works Ltd.

Alors que les Tribunaux américains avaient domé entièrement raison à la G. E. $\mathrm{C}^{\circ}$; à Londres, au contraire, le tribunal de Première instance et la Cour d'appel, tout en reconnaissant la brevetabilité et l'importance de premier ordre de l'invention, avaient statuŕ contre le brevet, dont les revendications (Claims) n'avaient pas paru répondre strictement aux conditions de pur formalisme exagérées par la législation anglaise des Brevets.

La British Thomson-Houston fit alors appel de larrêt devant la Chambre des Lords qui, statuant le 19 décembre dernier comme Cour Judiciaire Suprême, selon la Loi Anglaise, a réformé la décision des premiers juges et a prononcé, à l'unanimité et en derniere instance, la validité du brevet et la condamnation des contrefacteurs aux dépens des trois instances et ail paiement des dommages-intérêts, en réparation du préjudice causé aux propriétaires du brevet par la Corona Lamp Works Ltd du fait de ses contrefaçons.

Plus récemment, et à la suite de nombreuses contrefaçons du même genre, le même tébat a été engagé devant les tribunaux francais par la Compagnie des Lampes, à qui la Compagnie Française Thomson-Houston et la General Electric $C^{\circ}$ ont fait apport de tous leurs brevets visant les lampes à incandescence pour la France, l'Espagne, le Portugal, ainsi que les Colonies et pays de protectorat.

\section{Salon du Mobilier et des Arts appliqués.}

En plus du Congrès et du Concours de l'Hygiène, l'Office Central de la Construction et de l'Habitation ouvrira, au Palais de la Foire, du $1^{\text {er }}$ au 15 octobre 1922 , un Salon di Mobiluer et des Arts appliqués avec Concours de "Décoration moderne ", auquel des récompenses seront accordées aux lauréats.
Une section sera réservée aux Arts féminins (broderie, dentelles, cuir et métaux repoussés, pyrogravure, travaux d'étoffe, et $c .$. ).

Il y aura, en outre, une exposition rétrospective d'intérieurs et costumes de la région du sud-est (Bourgogne, Bresse, Provence, Auvergne, Savoie, etc..). 